\title{
ARTIGO 6
}

\section{MORTALIDADE INFANTIL EM UMA CAPITAL DO NORDESTE BRASILEIRO}

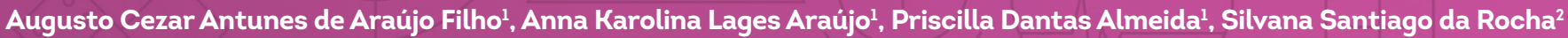

Objetivo: descrever a ocorrência da mortalidade infantil em Teresina (PI). Metodologia: estudo epidemiológico, descritivo, com dados secundários extraídos do Datasus, em que a população foi composta por todos os óbitos infantis, cujas mães são residentes em Teresina, no período de 2005 a 2014. Resultados: apesar das flutuações, houve declínio dos coeficientes de mortalidade neonatal, pós-neonatal e infantil. Houve predomínio de óbitos infantis em mães com idade entre 20 e 29 anos, e com escolaridade entre oito e 11 de estudo. Conclusão: existe a necessidade de melhorar políticas públicas e a assistência, embora tenha havido a redução da mortalidade infantil.

Descritores: Mortalidade infantil, Epidemiologia, Enfermagem.

\section{INFANT MORTALITY IN A NORTHEAST BRAZILIAN CAPITAL}

Objective: To describe the occurrence of infant mortality in Teresina (PI). Methodology: Descriptive epidemiological study with secondary data extracted from DATASUS, in which the population was composed of all infant deaths of mothers who are residents of Teresina, in the period from 2005 to 2014. Results: Despite fluctuations, neonatal, post-neonatal and infant mortality coefficients declined. There was a predominance of infant deaths in mothers aged between 20 and 29 years, and with education background between 8 and 11 grade. Conclusion: There is a need to improve public policies and assistance, even though there has been a reduction in child mortality.

Descriptors: Infant Mortality, Epidemiology, Nursing.

\section{LA MORTALIDAD INFANTIL EN UNA CAPITAL DEL NORDESTE DE BRASIL}

Objetivo: Describir la incidencia de la mortalidad infantil en Teresina (PI). Metodología: Estudio epidemiológico descriptivo con datos secundarios extraídos de DATASUS, en el que la población se compone de todas las muertes infantiles de las madres que residen en Teresina, en el período de 2005 a 2014. Resultados: A pesar de las fluctuaciones, neonatales, coeficientes de mortalidad post-neonatal e infantil se redujo. Hubo un predominio de las muertes infantiles en las madres de edades comprendidas entre los 20 y los 29 años, y con la educación entre ocho y 11 del estudio. Conclusión: Existe la necesidad de mejorar las políticas públicas y de asistencia, aunque se ha producido una reducción de la mortalidad infantil.

Descriptores: Mortalidad Infantil, Epidemiología, Enfermería.

${ }^{1}$ Enfermeiro(a). Mestre. Departamento de Enfermagem. Universidade Federal do Piaui, Brasil.

2Enfermeira. Doutora. Departamento de Enfermagem. Universidade Federal do Piaui, Brasil. E-mail: silvanasantiago27@gmail.com 


\section{INTRODUÇÃO}

A mortalidade infantil (MI) configura-se como um indicador sensivel às condições de vida e saúde de uma determinada população(1), pois as reflete diretamente, e permite estimar o risco de morte entre menores de um ano e expressar as iniquidades existentes nas diversas partes do mundo(2). Por isso, a redução desse indicador ainda se configura como um desafio para o sistema de saúde, e também para a sociedade(3).

Uma das principais metas de políticas públicas para a população infantil, nacionais e internacionais, é a diminuição da $\mathrm{MI}^{(4)}$. Nos anos 2000, o cenário internacional contou com o apoio da Organização das Nações Unidas que instituiu os Objetivos do Desenvolvimento do Milênio - os quais possuem como meta a redução de dois terços na mortalidade de crianças menores de cinco anos entre 1990 e 2015(2). A meta brasileira para 2015 - 15,7/1.000 Nascidos Vivos (NV) - foi cumprida em 2011, quando o Coeficiente de Mortalidade Infantil (CMI) do Brasil foi de 15,3/1.000 NV(2)

$\mathrm{Na}$ maioria dos paises, a mortalidade infantil está em declínio(5). No Brasil, não tem sido diferente, estudos demonstram uma tendência de redução em todas as regiões ${ }^{(4)}$. Entretanto, para atingir os niveis de regiões mundiais mais desenvolvidas, ainda existem alguns pontos que necessitam ser alcançados, como: a diminuição das desigualdades regionais e as iniquidades relacionadas a grupos sociais específicos(3), pois sabe-se que esse indicador apresenta uma grande variabilidade nas diferentes regiões do país ${ }^{(2.5)}$, muitas vezes, em um mesmo municipio(3).

No Brasil, o CMI caiu de 26,6 para 16,2/1.000 NV entre 2000 e 2010. Contudo, as desigualdades regionais continuaram existindo(5), pois a média do Brasil, em 2010 , foi de 16,0/1.000 NV, mas, nas regiões Norte e Nordeste, os coeficientes registrados foram de 21,0/1.000 NV e 19,1/1.000 NV, respectivamente(6).

Diante do exposto, torna-se fundamental analisar a MI nos diferentes contextos sociais brasileiros, a fim de fornecer subsídios para implantação de ações de saúde mais efetivas, com intervenções mais apropriadas às necessidades dos grupos populacionais de maior vulnerabilidade. Nesse sentido, o objetivo deste estudo é descrever a ocorrência da mortalidade infantil em Teresina (PI) no período de 2005 a 2014.

\section{METODOLOGIA}

Trata-se de um estudo epidemiológico, descritivo, com dados secundários coletados do Sistema de Informações sobre Mortalidade (SIM) e do Sistema de Informações sobre Nascidos Vivos (SINASC) do Ministério da Saúde, através do Departamento de Informática do Sistema Único de Saúde (DATASUS).

A população deste estudo foi composta por todos os óbitos infantis de mães residentes em Teresina no período de 2005 a 2014, disponíveis no site (www.datasus.gov.br). A cidade de Teresina é a capital do estado do Piauí, o qual está localizado no Nordeste do Brasil. Essa cidade tem uma extensão territorial de $1.391,981 \mathrm{~km}^{2}$, com população estimada para o ano de 2016, de 847.430 habitantes. Em 2010, o Índice de Desenvolvimento Humano Municipal (IDHM) foi de 0,751.

Os dados foram extraídos do DATASUS em dezembro de 2016, considerando-se as variáveis relacionadas aos óbitos infantis e aos nascidos vivos de Teresina-PI. Para calcular o CMI, dividiu-se o número total de óbitos de menores de um ano pelo número total de Nascidos Vivos (NV) de mães residentes na cidade, multiplicandose o resultado por 1.000. O cálculo do Coeficiente de Mortalidade Neonatal (CMN) deu-se por meio da razão entre o número de mortes de menores de 28 dias e o total de NV no mesmo ano, multiplicando-se o resultado por 1.000; e, para calcular o Coeficiente de Mortalidade PósNeonatal (CMPN), utilizou-se a razão entre o número de óbitos de crianças de 28 a 364 dias de vida e o total de NV no mesmo ano, multiplicando-se por 1.000. A tabulação dos dados ocorreu a partir do programa TABNET. Em seguida, os dados foram exportados para o EXCEL, programa em que se procedeu a análise.

Tendo em vista que os dados utilizados foram extraídos de uma plataforma de domínio público, este estudo não foi submetido ao Comitê de Ética em Pesquisa. Contudo, destaca-se que todos os aspectos, contidos na Resolução do Conselho Nacional de Saúde n. 466/12, foram observados.

\section{RESULTADOS}

Foram registrados, no SIM, 2.352 óbitos infantis de mães residentes em Teresina-PI no período estudado, sendo 1.736 neonatais, 616 pós-neonatais. O SINASC registrou 138.475 nascidos vivos no mesmo período. Durante a década analisada, o CMN foi de 12,5/1.000 NV, o CMPN foi de 4,4/1.000 NV e o CMI 17,0/1.000 NV.

O CMI teve um declínio de 19,9 em 2005, para 15,1/1.000 NV em 2014, representando um decréscimo de 24,1\%. O componente neonatal foi o principal responsável por essa diminuição, passando de 14,0 para 10,6/1.000 NV entre 2005 e 2014, representando uma redução de 24,3\%. O componente pós-neonatal teve declínio de $23,7 \%$, em que o coeficiente diminuiu de 5,9 para 4,5/1.000 NV (Figura 1). 
Figura 1. Coeficiente de mortalidade infantil, neonatal e pósneonatal de Teresina-PI, 2005-2014. Teresina, Piauí, Brasil, 2016 .

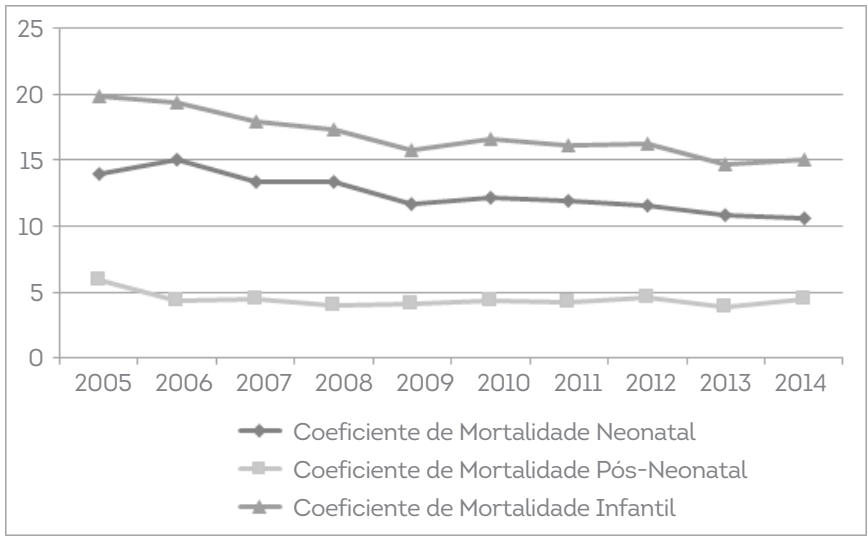

Fonte: Departamento de Informática do Sistema Único de Saúde (DATASUS), 2016.

Tabela 1 - Óbitos infantis segundo a idade e escolaridade maternas, duração da gestação e tipo de parto, no período de 2005-2014 em Teresina-PI. Teresina, PI, Brasil, 2016. $(\mathrm{n}=2.352)$

\begin{tabular}{|c|c|c|}
\hline Variáueis & $\mathrm{n}$ & $\%$ \\
\hline \multicolumn{3}{|c|}{ Idade da mãe (anos) } \\
\hline 10 a 14 & 36 & 1,53 \\
\hline 15 a 19 & 449 & 19,09 \\
\hline 20 a 29 & 1034 & 43,96 \\
\hline 30 a 39 & 472 & 20,07 \\
\hline 40 a 49 & 62 & 2,64 \\
\hline Ignorado & 299 & 12,71 \\
\hline \multicolumn{3}{|c|}{ Anos de escolaridade da mãe } \\
\hline Nenhuma & 100 & 4,25 \\
\hline 1 a 3 anos & 205 & 8,72 \\
\hline 4 a 7 anos & 518 & 22,02 \\
\hline 8 a ll anos & 727 & 30,91 \\
\hline 12 anos ou mais & 238 & 10,12 \\
\hline Ignorado & 564 & 23,98 \\
\hline \multicolumn{3}{|c|}{ Duração da gestação (semanas) } \\
\hline$<22$ & 125 & 5,31 \\
\hline 22 a 27 & 427 & 18,15 \\
\hline 28 a 31 & 476 & 20,24 \\
\hline 32 a 36 & 454 & 19,30 \\
\hline
\end{tabular}

\begin{tabular}{lcc|}
37 a 41 & 523 & 22,24 \\
\hline 42 ou mais & 25 & 1,06 \\
\hline Tipo de parto & 1171 & 49,79 \\
\hline Vaginal & 951 & 40,43 \\
\hline Cesáreo & 230 & 9,78 \\
\hline Ignorado & & \\
\hline
\end{tabular}

Fonte: Departamento de Informática do Sistema Único de Saúde (DATASUS), 2016.

Com relação ao peso ao nascer, observou-se maior frequência entre os recém-nascidos (RN) com baixo peso (35,08\%). Quanto à idade do óbito, houve maior concentração na primeira semana de vida (53,95\%). O hospital foi o local predominante de ocorrência de óbitos infantis $(94,22 \%)$ (Tabela 2).

Tabela 2 - Óbitos em menores de um ano segundo peso ao nascer, idade do óbito e local de ocorrência, no período de 2005-2014 em Teresina-PI. Teresina, PI, Brasil, 2016. $(\mathrm{n}=2.352)$

\begin{tabular}{|c|c|c|}
\hline Variáueis & $\mathbf{n}$ & $\%$ \\
\hline \multicolumn{3}{|l|}{ Peso ao nascer } \\
\hline Extremo baixo peso (< 1000g) & 705 & 29,97 \\
\hline Baixo peso (1000-2499g) & 825 & 35,08 \\
\hline Normo peso (2500-3999g) & 540 & 22,96 \\
\hline Macrossomia (4000g ou mais) & 36 & 1,53 \\
\hline Ignorado & 246 & 10,46 \\
\hline \multicolumn{3}{|l|}{ Idade do óbito (dias) } \\
\hline O a 6 dias & 1269 & 53,95 \\
\hline 7 a 27 dias & 467 & 19,86 \\
\hline 28 a 364 dias & 616 & 26,19 \\
\hline \multicolumn{3}{|l|}{ Local de ocorrência } \\
\hline Hospital & 2216 & 94,22 \\
\hline Domicilio & 114 & 4,85 \\
\hline Outros & 21 & 0,89 \\
\hline Ignorado & 1 & 0,04 \\
\hline \multicolumn{3}{|c|}{ Fonte: Departamento de Informática do Sistema Único de Saúde (DATASUS), 2016.} \\
\hline \multicolumn{3}{|l|}{ DISCUSSÃO } \\
\hline \multicolumn{3}{|c|}{$\begin{array}{l}\text { Embora com flutuações, o CMI apresentou redução no } \\
\text { ríodo estudado. A redução do CMI registrada corrobora }\end{array}$} \\
\hline
\end{tabular}


A queda da MI brasileira é relacionada às diversas melhorias nas condições de vida e de atenção à saúde da criança (como alimentação e nutrição, saneamento básico, vacinação e modelo de atenção à saúde)(3).

Apesar das flutuações em alguns anos, verificou-se redução de $24,3 \%$ e $23,7 \%$, durante a década analisada, nos componentes neonatal e pós-neonatal, respectivamente. Esse declínio também foi verificado em estudos realizados em Salvador-BA ${ }^{(7)}, 21,2 \%$ no $C M N$, e em Recife-PE ${ }^{(4)}$, decréscimo de $44 \%$ no $C M N$ e de $31,4 \%$ no CMPN. Isso evidencia melhoria na qualidade da atenção prestada no pré-natal, parto e ao RN na cidade de Teresina.

Neste estudo, identificou-se que a maioria dos óbitos infantis foi de mães com idade entre 20 e 29 anos, corroborando com estudos realizados em Porto Velho$\mathrm{RO}{ }^{(8)}$ e na Região Metropolitana de Maringá-PR( ${ }^{(9)}$, em que houve predomínio de mães com idade entre 20 e 34 anos.

A mortalidade neonatal,

componente da MI, associase à idade materna ao ter - filho, especialmente nos grupos etários extremos (adolescentes e mulheres acima de 35 anos), por causa de diversos fatores comportamentais, socioeconômicos e biológicos ${ }^{(8,10)}$

A maior ocorrência de óbitos infantis foi de mães com escolaridade entre 8 e 11 anos de estudo. Tendo em vista que o baixo grau de instrução da mãe é outro determinante da $\mathrm{MI}^{(11)}$, os achados deste estudo encontram-se divergentes aos da literatura. Em estudo realizado na Região Metropolitana de Maringá (PR), em que mães com menos de oito anos de estudo possuem mais chances de óbitos infantis $(\mathrm{OR}=1,4)^{(9)}$. Sabe-se que o aumento da escolaridade possui associação positiva com a redução da $\mathrm{MI}^{(12)}$, sendo considerada uma variável protetora entre a escolaridade materna superior a oito anos e a redução da $\mathrm{MI}^{(5)}$. Ademais, acredita-se que mães com um maior grau de instrução possuam melhores condições socioeconômicas e, consequentemente, maior acesso aos serviços de saúde, fatores que aumentariam a proteção ao óbito infantil(13).

Quanto à duração da gestação, houve predomínio do intervalo entre 37 e 41 semanas nos óbitos infantis, que contrapõe estudos desenvolvidos na Região Metropolitana de Maringá $(P R)^{(9)}$ e Foz do Iguaçu $(P R)^{(14)}$, os quais registraram que gestações com duração menor que 37 semanas possuem mais chances de óbitos infantis $(\mathrm{OR}=22,7)$ e $(O R=32,2)$, respectivamente. A sobrevivência dos recémnascidos cresce progressivamente quando a duração da gestação se distancia das 40 semanas $^{(15)}$

O baixo peso ao nascer é citado como fator de maior influência na determinação da morbimortalidade infantil(11), sendo considerado o principal preditor isolado da mortalidade infantil e neonatal ${ }^{(16)}$, existindo inclusive, uma relação inversamente proporcional entre mortalidade e peso ao nascer, em que a probabilidade de morte diminui à medida que o peso aumenta ${ }^{(10)}$. Neste estudo, a grande parte dos óbitos foi em RN com peso abaixo de 2.500 gramas, corroborando com estudo de Foz do Iguaçu (PR) ${ }^{(14)}$, no qual crianças nascidas com peso menor de $2.500 \mathrm{~g}$ possuem maior risco de óbito $(\mathrm{OR}=17,9)$. Entre os RN de muito baixo peso menos de $1.500 \mathrm{~g}$ - e os com $2.500 \mathrm{~g}$ ou mais, possuem risco de morte 30 vezes maior. Além do mais, os recém-nascidos com extremo baixo peso (peso menor $1.000 \mathrm{~g}$ ) possuem risco de óbito 4,8 vezes maior em relação aos com peso entre l.000g a $1.499 \mathrm{~g}^{(10)}$

A maioria dos óbitos foi registrada em recém-nascidos com 0 a 6 dias de vida, dados que corroboram com estudos realizados nas cidades de Ribeirão Preto-SP(3) e Porto Velho-RO(8). Identificou-se, também, que a maior parte aconteceu em hospitais, corroborando com estudo da Região Metropolitana de Maringá- $\mathrm{PR}^{(9)}$, em que 99,8\%

dos óbitos também ocorreram nesse cenário. Essas questões refletem, ainda, a necessidade da melhoria de atenção ao parto, nascimento e ao recém-nascido, ou seja, sugere a necessidade de reestruturação da rede cegonha, a qual deve ser integrada, hierarquizada e regionalizada, da ampliação da cobertura a fim de facilitar o acesso da população maternoinfantil e, ainda, da qualificação da assistência à gestante e ao recém-nascido para que ocorram avanços na redução da mortalidade infantil(17,8)

\section{CONCLUSÃO}

Apesar das reduções no coeficiente de mortalidade infantil e de todos os esforços para controlar esse indicador, ele ainda assola e constitui uma realidade negativa no Brasil e na capital do Piauí, Teresina, objeto deste estudo. A rede Cegonha trouxe diretrizes fundamentais para redução 
desse coeficiente, entretanto, ainda se faz necessário investimentos a fim de ampliar essas ações de saúde e obter resultados mais efetivos.

A saúde materno-infantil de Teresina, como no Brasil, ainda necessita de mais atenção e comprometimento de gestores e profissionais, os quais devem trabalhar em prol da mudança do paradigma atual, buscando aperfeiçoar a atenção durante o pré-natal, parto, nascimento e puerpério. Acredita-se que a melhoria nas políticas de saúde e execução das mesmas com compromisso constitui um aspecto relevante para mudar a atual situação de iniquidade.

\section{REFERÊNCIAS}

1. Nascimento SG, Oliveira CM, Sposito V. Ferreira DKS, Bonfim CV. Mortalidade infantil por causas evitáveis em uma cidade do Nordeste do Brasil. Rev Bras Enferm [Internet]. 2014 [citado 2016 dez 26]; 67(2): 208-12. Disponivel em: http://dx.doi.org/10.5935/0034-7167.20140027

2. Areco KCN, Konstantyner T, Taddei JAAC. Tendência secular da mortalidade infantil, componentes etários e evitabilidade no Estado de São Paulo - 1996 a 2012. Rev Paul Pediatr [Internet]. 2016 [acesso 2017 jan 03]; 34(3):263-270. Disponivel em: http://dx.doi.org/10.1016/j.rpped.2016.01.006

3. Careti CM, Scarpelini AHP, Furtado MCC. Perfil da mortalidade infantil a partir da investigação de óbitos. Rev. Eletr. Enf. [Internet]. 2014 [citado 12 dez 2016]; 16(2):352-60. Disponivel em: http://dx.doi.org/10.5216/ree. v16i2.20321

4. Cabral IC, Sousa MLXF, Dantas UIB, Reichert APS, Collet N, Santos SR. Mortalidade infantil: correlação entre indicadores de entes federativos nos anos de 2007 a 2011. Rev enferm UFPE on line [Internet]. 2013 [citado 2017 jan 03]; 7(9):5557-65. Disponivel em: http://www.revista.ufpe.br/ revistaenfermagem/index.php/revista/article/viewFile/4753/pdf_3407

5. Teixeira GA, Costa FML, Mata MS, Carvalho JBL, Souza NL, Silva RAR. Fatores de risco para a mortalidade neonatal na primeira semana de vida. Rev. pesqui. cuid. fundam. (Online) [Internet]. 2016 [citado 2017 jan 09]: 8(1):4036-4046. Disponivel em: http://dx.doi.org/10.9789/2175-5361.2016. v8il.4036-4046

6. Carvalho RAS, Santos VS, Melo CM, Gurgel RQ, Oliveira CCC. Desigualdades em saúde: condições de vida e mortalidade infantil em região do nordeste do Brasil. Rev. Saúde Pública [Internet]. 2015 [citado 2016 dez 26]; 49:5. Disponivel em:

http://dx.doi.org/10.1590/S0034-8910.2015049004794

7. Gonçalves AC, Costa MCN, Barreto FR, Paim JS, Nascimento EMR, Paixão ES et al. Tendência da mortalidade neonatal na cidade de Salvador (BahiaBrasil), 1996-2012. Rev. Bras. Saude Mater. Infant. [Internet]. 2015 [citado 2017 jan 12]: 15(3):337-347. Disponivel em: http://dx.doi.org/10.1590/S151938292015000300009

8. Moreira KFA, Oliveira TS, Gonçalves TA, Moura CO, Maluf SN, Tavares RSA et al. Mortalidade infantil nos últimos quinquênios em Porto velho, Rondônia - Brasil. Journal of Human Growth and Development [Internet], 2014 [citado 2017 jan 12]; 24(1): 86-92. Disponivel em: http://www.revistas. usp.br/jhgd/article/view/76123

9. Oliveira RR, Costa JR, Rossi RM, Rodrigues AL, Mathias TAF. Mortalidade infantil e fatores associados: estudo por tipologias sócio-ocupacionais. Cienc Cuid Saude [Internet]. 2015 [citado 2016 dez 26]; 14(2):1165-1174.
Disponivel em: http://ojs.uem.br/ojs/index.php/CiencCuidSaude/article/ view/24357

10. Gaiva MAM, Fujimori E, Sato APS. Mortalidade neonatal em crianças com baixo peso ao nascer. Rev Esc Enferm USP [Internet]. 2014 [citado 2017 jan 09]; 48(5):778-86. Disponivel em: http://www.scielo.br/scielo. php?script=sci_arttext\&pid=S0080-62342014000500778\&lng=en DOI: http://dx.doi.org/10.1590/S0080-6234201400005000002

11. Barbosa TAGS, Coelho KR, Andrade GN, Bittencourt DAS, Leal MC Gazzinelli A. Determinantes da mortalidade infantil em municipios do vale do Jequitinhonha, Minas Gerais, Brasil. Rev Min Enferm [Internet] 2014 [citado 20 dez 2016]; 18(4): 907-914. Disponivel em: http://dx.doi. org/10.5935/1415-2762.20140067

12. Faria R, Santana P. Variações espaciais e desigualdades regionais no indicador de mortalidade infantil do estado de Minas Gerais, Brasil. Saude soc. [Internet]. 2016 [citado $2016 \mathrm{dez} 26$ ]; 25(3):736-749. Disponivel em: http://dx.doi.org/10.1590/s0104-12902016147609

13. Rodrigues LS, Melo SF, Melo EVCF, Araújo A. Aspectos importantes sobre a mortalidade infantil em Itapecerica - Minas Gerais. R. Enferm. Cent. O. Min [Internet]. 2013 [citado 2017 jan 12]; 3(1):498-506. Disponivel em: http://www.seer.ufsj.edu.br/index.php/recom/article/viewFile/351/383

14. Doldan RV, Costa JSD, Nunes MF. Fatores associados à mortalidade infantil no Municipio de Foz do Iguaçu, Paraná, Brasil: estudo de casocontrole. Epidemiol. Serv. Saúde [Internet]. 2011 [citado 2017 jan 13]; 20(4):491-498. Disponivel em: http://dx.doi.org/10.5123/S167949742011000400008

15. Granzotto JA, Oliveira MB, Mendes RM, Winke S, Vecchi AA, Barros TP et al. Comportamento da mortalidade infantil no Extremo Sul do Rio Grande do Sul, Brasil, anos 2005-2012. Revista da AMRIGS [Internet]. 2014 [citado 2017 jan 13]; 58(2): 126-129. Disponivel em: http://www.amrigs.org.br/ revista/58-02/006.pdf

16. Silva CF, Leite AJM, Almeida NMGS, Leon ACMP, Olofin I. Fatores associados ao óbito neonatal de recém-nascidos de alto risco: estudo multicêntrico em Unidades Neonatais de Alto Risco no Nordeste brasileiro. Cad. Saúde Pública [Internet]. 2014 [citado 2017 jan 09]; 30(2):355-368. Disponivel em: http://dx.doi.org/10.1590/0102-311X00050013

17. Lansky S, Friche AAL, Silva AAM, Campos D, Bittencourt SDA, Carvalho ML et al. Pesquisa Nascer no Brasil: perfil da mortalidade neonatal e avaliação da assistência à gestante e ao recém-nascido. Cad. Saúde Pública [Internet]. 2014 [citado 2017 jan 09]; 30(Suppl 1): S192-S207. Disponivel em: http://dx.doi.org/10.1590/0102-311X00133213 